Please note this paper has been published ahead of print in Linguistic Approaches to Bilingualism: Digard, B. G., \& Sorace, A. (2021). Bringing together autism and bilingualism research: Language matters. Linguistic Approaches to Bilingualism. https://doi.org/10.1075/lab.21071.dig

Please do not cite this preprint. 


\title{
Bringing together autism and bilingualism research: language matters
}

\author{
Bérengère G. Digard \& Antonella Sorace \\ University of Edinburgh
}

(Commentary to keynote by Philippe Prevost and Laurice Tuller, "Bilingual language development in autism")

As pointed out by Prevost and Tuller (2022, hence P\&T), bilingual language development in autism is a recent field at the crossroad between autism and bilingualism research. Within the limited body of work already available, most publications focus on the language development of autistic bilingual children. ${ }^{1}$ Yet, even though this topic is addressed by most of the recent studies available, there are already a number of caveats and limitations that restrict the reproducibility and applicability of the findings.

As this field is relatively new, it is likely that many of its researchers approached the topic from either an autism or a bilingualism background, considering the other domain as a tool to learn more about their own topic of interest. As P\&T wrote in the opening of their keynote: "What can be learned about bilingualism by studying autism, and what can be learned about autism by studying bilingualism?". This approach, however, does not allow researchers, and by extension their studies, to fully grasp the ramifications of the other domain, be that autism or bilingualism. This may result in a body of work that does not actually acknowledge the full complexity of bilingualism in autism, and the richness of experiences and profiles existing within the autistic bilingual population. Indeed, as illustrated by the anecdotal cases reported by the authors, and as described in our recent publication on the language learning profiles and social experiences of 208 autistic bilingual adults (Digard et al., 2020), autistic children can become bilingual in as many ways as their neurotypical peers, through fairly common journeys and unusual circumstances alike. In sum, bilingualism in autism is at the opposite of a one size fits all.

This reality means that the field should move away from simply using either autism or bilingualism as a tool to understand the other type of experience. It is time to fully map out the specificities and complexities of this field on its own terms, genuinely bringing together autism and bilingualism research in an integrated manner. This change in approach will allow for robust research driven by community needs and preoccupations, grounded in relevant theories, accounting for its full complexity, and producing meaningful results that will positively impact bilingual people and their communities.

As a first step in this endeavour, the authors make a number of highly relevant points. Indeed, this review builds upon previous reviews on the topic by focusing on a critical assessment of the methods used in the available body of work. The authors rightfully argue that the tasks routinely used to measure language skills in autistic children are not suitable to produce an accurate picture of the children's skills, strengths, and difficulties alike, especially when they are growing up in a bilingual environment. Instead, there is a need for data on a wider range

\footnotetext{
${ }^{1}$ In this commentary we use identity-first language (e.g. "autistic people") as this is the preferred language of many people in the autistic and autism communities (Kenny et al., 2016; Sinclair, 2013).
} 
of linguistic and non-linguistic skills in autistic bilingual children, and future studies should make sure to adapt the level of details needed to comprehensively address their research question, avoiding the reliance on a single score task by default.

The authors are also correct to argue that a categorical approach of bilingualism is at best unhelpful to understand the effects of bilingualism in autism. Instead, the field needs to adopt a more ecologically valid definition of bilingualism that will allow us to understand the role of different aspects of the bilingual experience, such as age of onset, exposure, proficiency, dominance, use (especially in therapy), or language distance. As recommended by P\&T, richer and more meaningful reporting and characterisation of the autistic bilingual children taking part in the studies will be critical to better understand the interplay between bilingualism and autism in some specific contexts, such as language regression, language impairment, or learning disability. If anything, adequate characterisation of the samples will at least highlight the dearth of data regarding these specific children. This detailed level of understanding will allow researchers and practitioners to offer advice and support tailored to the specific needs and profile of each autistic bilingual person (Davis et al, 2021).

However, we argue that shift must not only come in the methods, but also in researchers' attitude, leading to a change of both substance and form. Future research should be scientifically grounded in adequate methods, of course, but also created in partnership with stakeholders from the autistic bilingual community and its allies, and shared in a respectful manner, both within and outwith academia. This also entails adopting the appropriate language when writing about autism and autistic people in academic publications. Just as for other research with non-neurotypical populations, researchers must earn the trust of the autistic bilingual community, which cannot be achieved when researchers use terms such as "severity", "affected", "spared", or "normal" when other more appropriate terms could as efficiently convey the information. Focusing on a niche and recent topic cannot be an excuse for the use of inappropriate terminology, such as some of the terms used by the authors of this keynote - i.e. "there is a great heterogeneity as to which language domain are affected/spared" (page 2) "Normal bilingual language acquisition" (page 2), "more severe autistic symptoms" (page 4), or "autism severity in bilingual children with ASD" (title of section 4.4.2, page 15).

Indeed, this specific point may be especially critical in autism research in general, and in the niche topic of bilingualism in autism in particular. Neurodiversity and autism pride movements are now successfully reshaping how researchers talk about autism in their own terms (Dyck \& Russell, 2020), but this shift towards more respectful language might be happening more slowly in countries and cultures with a more deficit-based and stigmatising attitude regarding autism than that we are observing in the UK or in some parts of North America (Cascio, 2015). However, the advances needed in the field of bilingualism in autism require international collaboration, and researchers from countries that have not yet widely adopted the appropriate language regarding autism should also make this additional shift in attitude, and be active allies of neurodiversity pride movements in their own countries. Without this shift, many valuable concepts and findings might find themselves lost in translation. 
The research available to date does suggest that in general at least, a bilingual upbringing is not detrimental for autistic people, but we are still in the dark regarding the connections between specific autism profiles, specific bilingual experiences, and specific linguistic or cognitive skills. When it comes to the experiences of autistic bilinguals, almost everything has to be properly understood. This new research field has its whole history left to write, and we researchers must work together to ensure it is built in a robust and respectful way, promoting participatory, reproducible, and suitable methods that acknowledge and support the great diversity of autistic bilinguals. This global approach cannot be only limited to a more adequate choice of tasks, but also extended to the words used to report the results of these tasks. In the field of bilingualism in autism as in any other, and maybe more than in any other, language matters.

\section{References}

Cascio, M. A. (2015). Cross-Cultural Autism Studies, Neurodiversity, and Conceptualizations of Autism. Culture, Medicine, and Psychiatry, 39(2), 207-212. https://doi.org/10.1007/s11013-015-9450-y

Davis, R., Fletcher-Watson, S., \& Digard, B. G. (2021) Autistic People's Access to Bilingualism and Additional Language Learning: Identifying the Barriers and Facilitators for Equal Opportunities. Frontiers in Psychology, 12, 741182. http://doi.org/10.3389/fpsyg.2021.741182

Digard, B. G., Sorace, A., Stanfield, A., \& Fletcher-Watson, S. (2020). Bilingualism in autism: Language learning profiles and social experiences. Autism, 1362361320937845. https://doi.org/10.1177/1362361320937845

Dyck, E., \& Russell, G. (2020). Challenging Psychiatric Classification: Healthy Autistic Diversity and the Neurodiversity Movement. In S. J. Taylor \& A. Brumby (Eds.), Healthy Minds in the Twentieth Century: In and Beyond the Asylum (pp. 167-187). Springer International Publishing. https://doi.org/10.1007/978-3-030-27275-3_8

Kenny, L., Hattersley, C., Molins, B., Buckley, C., Povey, C., \& Pellicano, E. (2016). Which terms should be used to describe autism? Perspectives from the UK autism community. Autism, 20(4), 442-462. https://doi.org/10.1177/1362361315588200

Prévost, P., \& Tuller, L. (2022). Bilingual language development in autism. Linguistic Approaches to Bilingualism, 12(1).

Sinclair, J. (2013). Why I dislike "person first" Language. Autonomy, the Critical Journal of Interdisciplinary Autism Studies, 1(2), Article 2. http://www.larryarnold.net/Autonomy/index.php/autonomy/article/view/OP1 